\title{
Mucormicosis Maxilar posterior, secundaria a una exodoncia: Reporte de un caso y revisión de la literatura.
}

\section{Posterior maxillary mucormycosis, secondary to an exodontics: A case report and literature review.}

\author{
Francisco Yévenes-Souper ${ }^{1 *}$, Marlene Díaz-Reiher ${ }^{1}$, María de los Ángeles Fernández-Toro", \\ Francisca Donoso- Hofer ${ }^{1}$.
}

\author{
1. Cirugía y Traumatología Maxilofacial, Servicio \\ Dental, Hospital San Juan de Dios, Santiago, Chile. \\ * Correspondencia Autor: Francisco Yévenes- \\ Souper | Servicio de Cirugía y Traumatología \\ Maxilofacial, Hospital San Juna de Dios | \\ Dirección:Av. Portales \#3239, Santiago, \\ Chile. | Teléfono: +(56) 9 97135940. | E-mail: \\ fyevenessouper@gmail.com \\ Trabajo recibido el 24/03/2020. \\ Trabajo revisado 10/09/2020 \\ Aprobado para su publicación el 25/09/2020
}

\begin{abstract}
RESUMEN
La Mucormicosis se describe como una infección fúngica de tipo oportunista y potencialmente mortal, reportándose en la literatura como la tercera en frecuencia, muy por detrás de la Candidiasis y Aspergilosis. Esta infección es causada por hongos de la familia de los Mucorales. Se presenta el caso de un hombre con Diabetes Mellitus II con un control metabólico deficiente, el cual posterior a una exodoncia, presenta una Mucormicosis Maxilar, presentando una etiología atípica y logrando ser confirmada posterior a biopsias y pruebas de cultivo, demostrando elementos del tipo Mucor. Se realizó una Maxilectomía parcial del lado afectado como tratamiento con una evolución favorable. Esta revisión destaca la importancia de la búsqueda activa basada en la semiología y la importancia de los exámenes complementarios, implicando lograr un correcto diagnostico y pronostico de la enfermedad.
\end{abstract}

PALABRAS CLAVE:

Mucormicosis; Osteomielitis; Hifas; Inmunocomprometido.

Int. J. Inter. Dent Vol. 14(3); 271-273, 2021.

\section{ABSTRACT}

Mucormycosis is described as an opportunistic and life-threatening fungal infection, being reported in the literature as the third in frequency, far behind candidiasis and aspergillosis. This infection is caused by fungi of the Mucorales family. We present the case of a man with Diabetes Mellitus II with poor metabolic control who, after an extraction, presents a Maxillary Mucormycosis, with an atypical etiology and confirmed after biopsies and culture tests, demonstrating elements of the Mucor type. A partial maxillectomy was performed on the affected side as a treatment with a favorable outcome. This review highlights the importance of active search based on semiology and of complementary examinations, implying a correct diagnosis and prognosis of the disease.

KEY WORDS:

Mucormycosis; Osteomyelitis; Hyphae; Immunocompromised.

Int. J. Inter. Dent Vol. 14(3); 271-273, 2021.

\section{INTRODUCCIÓN}

La Mucormicosis se describe como una infección fúngica de tipo oportunista y potencialmente mortal, reportándose en la literatura como la tercera en frecuencia, muy por detrás de la Candidiasis y Aspergilosis, en donde las series extranjeras informan de 1,7 casos por cada 1 millón de habitantes ${ }^{(1,2)}$. Esta infección es causada por hongos de la familia de los Mucorales, donde se encuentran entre los más frecuentes, Mucor, Absidia Rhizomucor, y Rhizopus, siendo este último el agente etiológico más común en una Mucormicosis en humanos ${ }^{(3)}$.

Debido al aumento de la esperanza de vida, la incidencia de la Mucormicosis ha ido en aumento, en donde, los principales hospederos en riesgo son los pacientes inmunocomprometidos, entre los que se encuentran aquellos individuos con Diabetes Mellitus (DM), Leucemia, Cáncer, Trasplantados de Órganos e inmunosuprimidos por fármacos. Se observa una predisposición en los pacientes diabéticos (36-88\% de los casos de Mucormicosis), especialmente en aquellos que no presentan un adecuado control metabólico. La presentación de la mucormicosis en estos pacientes es rápida, fulminante y letal ${ }^{(4,5)}$.

Se presenta el caso clínico de un paciente con Mucormicosis Maxilar con una semiología atípica, discutiéndose la relevancia de realizar un buen diagnóstico diferencial para así tratar oportunamente esta grave patología que, de lo contrario, puede resultar mortal.

Este reporte de caso fue preparado siguiendo las directrices $\mathrm{CARE}^{(1)}$.

\section{PREESENTACIÓN CASO}

Paciente género masculino, de 55 años, ingresa al Servicio de Cirugía y Traumatología Máxilofacial del Hospital San Juan de Dios, Santiago de Chile, refiriendo que fue sometido a exodoncia de molar maxilar derecho hace 2 meses, que evolucionó con exudado purulento y dolor de tipo pulsátil, de intensidad EVA 8/10, intermitente, localizado en hemimaxilar derecho irradiado a región cigomática y palatina ipsilateral, que no cede frente a analgésicos. Acude con drenaje tipo Penrose y con antibioterapia empírica con Azitromicina y Metronidazol 500/500 mg ya iniciada evolucionando tórpidamente.

Dentro de la anamnesis remota se constata Diabetes Mellitus II con control metabólico intermitente de 8 años de evolución, en tratamiento con Metformina $850 \mathrm{mg} 3$ veces/día, Glibenclamida $5 \mathrm{mg} 1$ vez/día, Insulina $\mathrm{NPH} 10 \mathrm{UI} 2$ veces/día; Hipertensión Arterial en tratamiento diario con Enalapril $10 \mathrm{mg}$ y Aspirina $100 \mathrm{mg}$. No refiere otros antecedentes mórbidos 


\section{relevantes.}

Al examen físico se observó aumento de volumen hemifacial derecho con borramiento del surco nasogeniano ipsilateral. Intraoralmente, se observó exudado purulento en sitio de exodoncia y a través de drenaje palatino, comunicación oro-antral y ósea al medio bucal de aspecto necrótico (Figura 1).

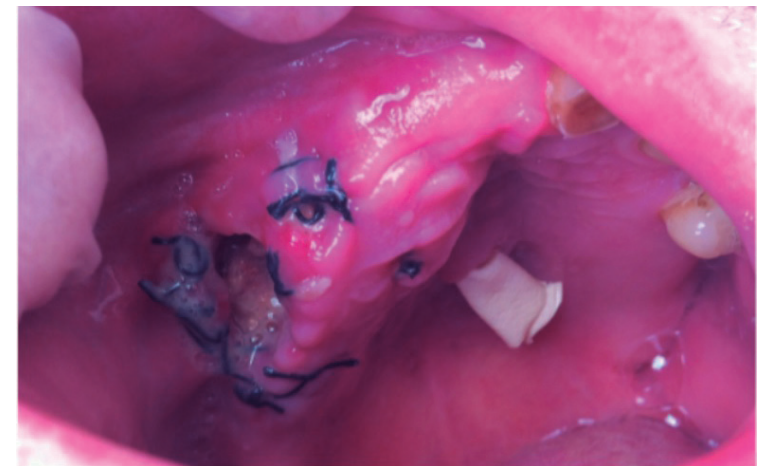

Figura 1. Aspecto de cavidad intra oral del paciente.

La Tomografía axial Computada (TAC) reveló una sinusitis hemimaxilar y etmoidal derecha sumado a una lesión osteolítica ipsilateral que compromete hasta el piso de la órbita (Figura 2).

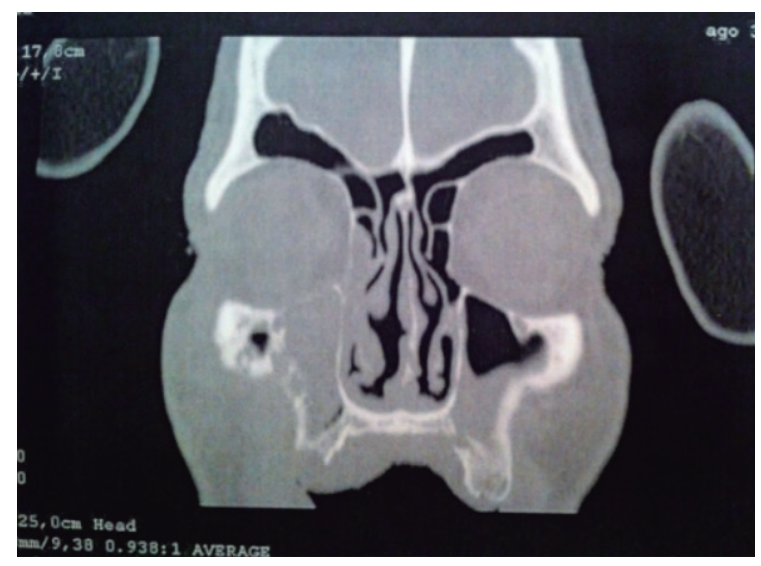

Figura 2. TAC. Corte Coronal. Se observa engrosamiento sinusal y compromiso seno maxilar derecho.

\section{Tratamiento Médico:}

Al iniciar hospitalización para estudios del paciente, se cambia esquema antibiótico por Penicilina Sódica 5 millones UI en conjunto con AINES. Se realizó, además, una primera prueba para cultivo de microorganismos del contenido purulento, expresando como resultado para la Tinción Gram: Cocaceas gram (+) escasas; Bacilos gram (+) escasos y Polimorfonucleares en cantidad regular; procediendo posteriormente a realizar un aseo quirúrgico de la zona, biopsia incisional y segunda prueba de cultivo.

El diagnóstico histopatológico correspondió a numerosas Hifas no septadas del tipo Mucor y Osteomielitis. El paciente se mantuvo en control constante por el equipo interdisciplinario decidiéndose iniciar tratamiento antibiótico con Anfotericina B 50 mg EV.

El segundo cultivo resultó positivo para Hifas gruesas aseptadas, levaduras y Cándida Albicans en tejido óseo.

\section{Tratamiento Quirúrgico:}

Se realizó una resección quirúrgica con abordaje de Weber y Fergusson, disección Nasogeniana lateral hasta exposición amplia de plano óseo comprometido con lesión, encontrando, además, compromiso malar, de arco cigomático, piso orbitario en su segmento medial (Figura 3). Por consiguiente, se decidió realizar una Maxilectomía subtotal derecha y una cobertura de la cara medial del colgajo miocutáneo con injerto dermoepidérmico tomado de muslo izquierdo. En la biopsia diferida tomada en el acto quirúrgico se describió la presencia de elementos con morfología Mucor a nivel de tejido óseo y proceso inflamatorio crónico activo granulomatoso.

Se presentan imágenes de controles correspondientes al primer y tercer mes (Figura 4 y 5) post quirúrgico y de tratamiento con Anfotericina B en donde se observa una evolución favorable.

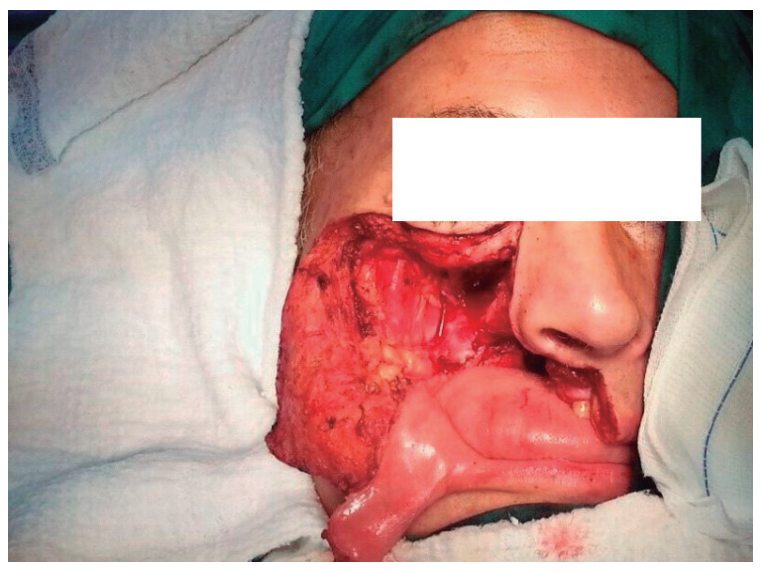

Figura 3. Abordaje de Webber y Fergusson y Maxilectomía derecha.
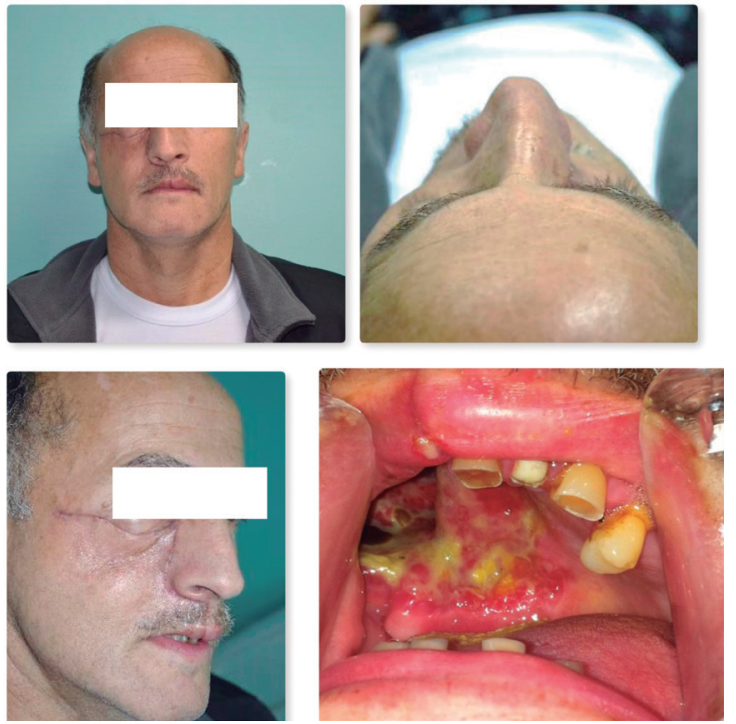

Figura 4. Múltiples vistas de la evolución del paciente en su primer mes post operatorio. Se observan vistas extraoral e intraoral.

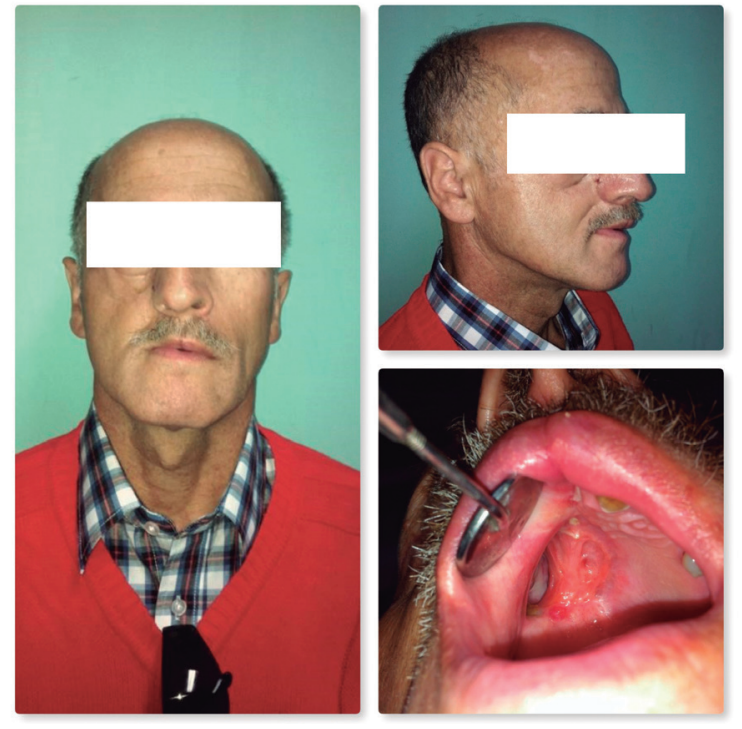

Figura 5. Múltiples vistas de la evolución del paciente en su tercer mes post operatorio. Se observan vistas extraoral e intraoral de su evolución favorable.

\section{DISCUSIÓN}

La extracción dental o exodoncia es un procedimiento rutinario en la práctica odontológica, desarrollada en la mayoría de los casos sin complicaciones post operatorio. 
Sin embargo, en aquellos pacientes que presentan inmunosupresión u otras alteraciones sistémicas, como la Diabetes Mellitus, pueden producirse ciertos desórdenes y alteraciones en la cicatrización de la mucosa y tejido óseo; aspectos que predisponen la instalación de procesos patológicos, como una Osteomilitis o una Mucormicosis maxilar.

El caso presentado plantea una situación poco común y bastante relevante: la disminución del aporte vascular e inoculación de microrganismos, cuyo factor común es el compromiso sistémico del paciente, traducido en un cuadro de Mucomicosis y Osteomielitis(6,7).

La Mucormicosis es descrita como una infección oportunista grave, producida por hongos, infrecuente y con un gran potencial agresivo y fatal(4,1). Produce como alteración más significativa los infartos tisulares y se describe como la tercera Micosis más invasiva en frecuencia. Existen distintas formas clínicas aceptadas, entre las que se encuentran la presentada, con inicio en el seno maxilar y posterior compromiso Rinoórbito-cerebral $(8,9,10,11)$.

En el caso presentado, secundario a una exodoncia maxilar el paciente cursó con una comunicación oro antral un proceso infeccioso y necrótico activo -como la Osteomielitis-, lo que genera las condiciones que favorecen la instalación de una Mucormicosis ${ }^{(12)}$. Sumado a lo anterior, la descompensación metabólica del paciente implica capacidades fagocíticas, leucocitarias y quimiotácticas disminuidas, lo cual predispone en un $70 \%$ a contraer enfermedades oportunistas.

La gran cantidad de cuerpos cetónicos existentes, elevan los niveles de hierro sérico; lo que, sumado a la acidificación del medio, provoca un desplazamiento en la unión del Hierro con su trasportador (Transferrina) que permite la captación por parte del hongo, aumentando así la posibilidad y riesgo de desarrollar infecciones fúngicas invasivas. Por su parte, el hongo aumenta la producción de una enzima llamada Cetoreductasa, que actúa sobre estos cuerpos cetónicos propiamente tales, provocando que el ambiente sea cada vez más ácido, lo que favorece la prevalencia de los mucorales ${ }^{(11,13)}$, se hace presente que este último proceso, no es del todo claro.

Debido a que la tasa de mortalidad global de la Mucormicosis es del $44 \%$ en pacientes diabéticos en condiciones como las presentadas en este caso(4), se debe poner especial énfasis en una correcta evaluación orientadas hacia la patología específica.

Mediante exámenes de laboratorio, biopsias, pruebas de cultivo, sumado a pruebas histoquímicas como la tinción de PAS; se pudo cuantificar y determinar desde el estado de descompensación sistémica del paciente sumado a la presencia de un proceso infeccioso e inflamatorio hasta por medio de la tinción de PAS ya mencionada, exponer diagnósticos diferenciales con otros tipos de hongos filamentosos como Aspergillus spp, Fusarium spp y Pseudallescheria boydii(14) (figura 6).

En términos generales, existen tres pilares fundamentales como terapia para esta infección. En primer lugar, se postula el tratamiento y compensación de la enfermedad base en un ambiente hospitalario, en este caso Diabetes. Como segundo pilar, iniciar una terapia antifúngica sistémica, siendo la más ampliamente aceptada aquella que fue utilizada en este caso (Anfotericina B), y evaluar de igual forma si es necesario el uso de fármacos sinérgicos. Tercero, es la cirugía resectiva, agresiva y precoz, con remoción de todo el tejido infectado y debridación ${ }^{(2,3)}$.

La Maxilectomía subtotal derecha se realizó con el fin de eliminar la contaminación y difusión del proceso infeccioso hacia estructuras que puedan comprometer la vida del paciente.

De manera general y al igual que en el caso presentado, en los cuadros de Mucormicosis, la ausencia de signos clínicos patognomónicos y la imperiosa necesidad de realizar un diagnóstico histopatológico hace

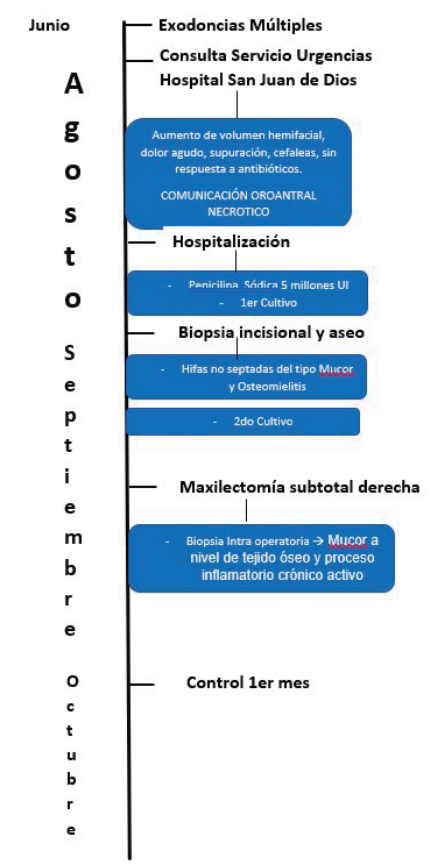

Figura 6. Cronograma de intervenciones y resultados a propósito del manejo y estudio de Mucormicosis.

fundamental que en los pacientes que presenten condiciones de riesgo se realice un estudio dirigido y específico.

\section{CONCLUSIÓN}

La Mucormicosis es una infección fúngica agresiva con una alta tasa de mortalidad, especialmente en pacientes inmunocomprometidos, donde hay múltiples factores que son relevantes como la inmunosupresión del paciente y evolución de la pato fisiología de la infección fúngica frente a Osteomielitis de los maxilares.

En el caso de las complicaciones sinusales, puede llegar a ser fatal el compromiso de ciertas estructuras como órbita, seno cavernoso y cerebro, por lo que el diagnóstico temprano es imperativo pata el comienzo oportuno del tratamiento, mejorando la posibilidad de sobrevida del paciente.

\section{RELEVANCIA CLÍNICA}

La Mucormicosis como patología fúngica presenta una gran agresividad y a la vez una alta tasa de mortalidad, se presenta como una enfermedad oportunista, muchas veces silente y con una presentación atípica. Se describe los aspectos relevantes de la patología y cómo poder llevar un tratamiento adecuado para conservar la vida del paciente, esperando sea de ayuda a las distintas especialidades a la hora del diagnostico

\section{CONFLICTO DE INTERESES}

Los autores no presentan conflicto de interés para este artículo

\section{Bibliografía}

1. Eucker J, Sezer O, Graf B, Possinger K. Mucormycoses. Mycoses. 2001;44(78):253-60.

2. Rahal M, Moreno M, Villa J. Mucormicosis rino-orbito-cerebral: Experiencia en doce años. Rev Otorrinolaringol Cir Cabeza Cuello. 2008;68(1):27-34.

3. Spalloni P, Chavez A, Aviles C, Cofré J. Mucormicosis en pediatría. Rev Chil Infectol. 2004;21(1):17-25

4. Sujatha SR, Chauhan $P$, Sharma $S$. Rhinocerebral mucormycosis among diabetic patients: an emerging trend. Mycopathologia. 2015;180(5-6):389-96.

5. Irga N, Kosiak W, Jaworski, Komarnicka, Birkholz. Hyperthyroidism secondary to disseminated mucormycosis in a child with acute lymphoblastic leukemia: case report and a review of published reports. Mycopathologia. 2013;175(1):123-7.

6. Kremers HM, Nwojo ME, Ransom JE, Wood-Wentz CM, Melton III LJ, Huddleston III PM. Trends in the epidemiology of osteomyelitis. J Bone Joint Surg Am. 2015;97(10):837-45.

7. Strumas N, Antonyshyn O, Caldwell CB, Mainprize J. Multimodality imaging for precise localization of caniofacial osteomyelitis. J Craniofac Surg. 2003;14(2):215-19.
8. Prabhu R, Patel R. Mucormycosis and entomonphthoramycosis a review of the clinical manifestations diagnosis and treatment. Clin Microbiol Infect. 2004;1(1):31-47. 9. Ventura GJ, Kantarijan H, Anaissie E, Hopfer R, Fainstein V. Pneumonia with Cunninghamekka species in patients with hematologic malignancies: a case report and review of the literature. Cancer. 1986;58(1):1534-6.

10. Pincus DJ, Armstrong MB, Thaller SR. Osteomyelitis of the Craniofacial Skeleton. Semin Plast Surg. 2009;23(2):73-9.

11. Urs A, Singh H, Monhanty S, Sharma P. Fungal osteomyelitis of maxillofacial bones: rare presentation. J Oral Maxillofac Pathol. 2016;20(3):546.

12. Chakrabarti A, Das A, Mandal J, Shivaprakash MR, George VK, Tarai B, et al. The rising trend of invasive mucormycosis patients with uncontrolled diabetes mellitus. Med Mycol. 2006; 44(4):335-42.

13. Alvarez F, Fernandez-Ruiz M, Aguado JM. Iron and Invasive fungal infection. Rev Iberoam Micol. 2013;30(4):217-25

14. Tapia O, Chahín C, Concha C. Mucormicosis cutánea primaria: a propósito de dos casos. Revisión de la literatura. Rev Chil Infectol. 2011;28(3):269-73. 